\title{
Mathematical Models for Calculation of Mixing Zones for Coastal Effluent Discharges
}

\author{
Anton Purnama, Huda A. Al-Maamari, Easwaran Balakrishnan
}

\begin{abstract}
The potential long-term environmental impacts of coastal effluent discharges can be addressed and regulated using a mixing zone concept to control and manage the mixing capacity of the receiving coastal waters. The standard regulatory guidelines consist of two key elements: a concentration limit and a point of compliance expressed as a radius centered at the end of the outfall pipe. Modeling studies of the effect of seabed depth upon dispersion of coastal effluent discharges into the sea in the far-field are investigated analytically using the solutions of two-dimensional advection-diffusion equations with a point source on the simple depth profiles of a flat seabed and a uniformly sloping seabed. Solutions are then illustrated graphically by plotting contours of concentration, showing that the effluent discharged plumes are spreading downstream and heading towards the shoreline. Based on the location of maximum value of concentration at the shoreline, the imposed radius of and limit of concentration level within the circular mixing zone are formulated to be used as a measure for assessing the impact of effluent discharges in the coastal environment. The results found agreed with the mixing zones implemented in practice as the environmental quality standards by the regulatory authorities to minimize the impact. As the sea outfall's long pipeline may go beyond the continental shelf, a sudden step change in the seabed depth profiles is introduced, where the solutions of the advection-diffusion equations are obtained using the method of images. An extended formulation of the mixing zones radius and concentration limit are also presented.
\end{abstract}

Index Terms: advection diffusion equation, flat seabed, sloping seabed, water quality model.

\section{INTRODUCTION}

For coastal industrial plants, it is a safe, economical and reliable solution to discharge pretreated effluent wastewater into the sea through an effective, well-designed outfall system [1], [2]. A long sea outfall is a pipeline constructed for discharging effluent streams into the ocean. Extensive experiences around the world have reported that an outfall system installed with multiport diffusers has minimal environmental impacts since it apparently prevents the effluent discharged plumes from reaching coastal areas of human usage [1], [2]. For the near-shore discharges, it is observed in the far-field that the bent-over effluent plumes

\footnotetext{
Revised Manuscript Received on September 22, 2019.

Anton Purnama, Department of Mathematics, Sultan Qaboos University, College of Science, Muscat, Sultanate of Oman.

Huda A. Al-Maamari, Department of Mathematics, Sultan Qaboos University, College of Science, Muscat, Sultanate of Oman.

Easwaran Balakrishnan, Department Mathematics, Sultan Qaboos University, College of Science, Muscat, Sultanate of Oman.

This work was supported by the Sultan Qaboos University Internal Grant IG/SCI/DOMS/18/04
}

are spreading towards the shoreline and may impair coastal water quality and affect marine life, and may also create a health concern to the public who use the nearby beaches for swimming and other recreational purposes [3], [4]. The extent and magnitude of changes in the receiving water quality due to effluent discharges define a mixing zone. Thus, the main objective of the coastal discharges is to meet the water quality standards set at a certain safety level (within a prescribed circular mixing zone) by the regulatory authorities to protect the receiving coastal and marine environment and human health [1], [4]-[6].

A regulatory mixing zone (RMZ) is an allocated impact zone in the receiving waters around the outfall beyond which the imposed water quality criteria must be met [4], [5], [7]-[9]. Conventionally, this standard regulatory guidelines consists of a concentration limit $C_{*}$ and a point of compliance expressed as a distance ${ }^{R *}$ from the outfall's discharge port. In order to minimize the impacts of coastal effluent discharges, the mixing zones should be kept to the smallest area as practicable. It is recommended that the excess concentration limit at the mixing zone boundary normally should not exceed $5 \%$ (or correspond to a minimum dilution of about 20:1) of that occurring naturally in the waters around the discharge within a radius $R_{*}$ that varies from 50 to $300 \mathrm{~m} \mathrm{[4],} \mathrm{[7],} \mathrm{[10].} \mathrm{Note} \mathrm{that} \mathrm{for} \mathrm{acutely} \mathrm{toxic}$ effluents discharge, both the allowable concentration limit and mixing zone size are smaller than that of the specified values for conventional effluents.

The field monitoring and predictive models are widely used in order to demonstrate the compliance of operating an outfall system to discharge effluents with the regulatory standards. Due to a unique nature and local conditions of the receiving water, no unified method can be used to establish a mixing zone, and each discharge should be analyzed separately on a case-by-case basis. Thus, no specific mathematical models are recommended to assess the dilution and dispersion capacities of the receiving waters, and in searching for baseline quality standards for sophisticated, computational (full) three-dimensional hydrodynamic and water quality models, the analytical solutions of simple far-field models are presented to perform preliminary worst-case assessments [7]-[9], [11].

Many large cities are located on the coastal areas, and many operated outfall systems are built predominantly on sandy beaches. Physically, the coastal area is a dynamic region where land and sea meet. In some places, it takes the form of a sloping sandy beach, but in other places it is a mountainous coast with rocky sea cliffs, where the water depth gets deep very rapidly

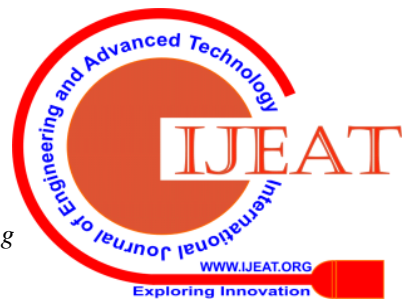


within a short offshore distance [3], [12], [13]. There are no beaches with seabed's sloping uniformly towards the sea. Going further seaward from the shore, the first submerged region is termed continental shelf. The seaward limits of the shelf are determined by the distinct change in slopes between the shelf and its adjacent continental slope. Thus a seabed depth profile is typically depicted in the oceanography textbooks as a gentle slope coming in contact with a steeper one.

To study the effect of water depth variations upon dispersion of coastal effluent discharges into the sea, we first consider simple profiles of a flat seabed with a constant water depth and a uniformly sloping seabed [14]-[16]. Solutions of a two-dimensional advection-diffusion equation with a point source are presented graphically by plotting contours of concentration showing that the discharged effluent plumes are spreading downstream towards the shoreline. However, it seems impractical to impose the concentration limit value obtained at the shoreline to the operators of marine outfall systems. The difficulties in adopting a mixing zone concept are mainly related to setting up the concentration limit $C_{*}$ to be met at the circular mixing zone boundary of radius $R_{*}$ [5], [8], [9], [11]. We proposed to use the location of the maximum value of concentration at the shoreline to set up the radius (from the point source) of the RMZ and then use it to formulate the concentration limit. Finally, using the method of image, the solutions are extended to a point discharge on a step change in seabed depth profiles formed by joining a shallow flat seabed to a deeper flat seabed and, for a sloping seabed, to another sloping seabed with a steeper slope [13].

\section{Model Solutions For CoASTAL Discharges ON CONTINUOUS SEABED DEPTH}

Modeling the environmental impact of effluent discharges into the sea is a difficult and complex task due to the variety and variability of the mixing processes, and the change in the sea currents, temperature and density. Conventionally, a local mixing zone should be determined based on the worst-case scenario of calm sea conditions drifting with a steady current. As we are only concerned with the effect of the seabed depth profile (perpendicular to the coastline), for simplicity, the other complexities such as tidal motions, temperature and density are ignored. The sea is assumed to be wide, the shoreline straight, and the effluent is discharged with a rate $Q$ from a point source (from the end of outfall pipe) at $\left(0, \alpha h_{0}\right)$, where $h_{0}$ is an arbitrary reference water depth. The marine outfall systems are commonly installed with multiport diffusers designed to rapidly mix and dilute the discharged effluents with the receiving sea currents. It is also assumed that the effluent concentration is vertically well-mixed over the water depth.

As waves at sea approach the beach at an angle, the shallow seabed refracts and generates the (net coast parallel-component) longshore currents. The current is assumed to be steady with speed $U$ and remain in the $x$-direction (positive to right of the outfall) at all times. The dispersion processes are represented by eddy diffusivities, and diffusion in the $x$-direction is neglected, as the

discharged plumes in steady currents become very elongated in the $x$-direction. The variations in the $y$-direction (positive towards the sea) of current speed $U$ and coefficient of diffusivity $D$ are assumed as the power functions only of water depth $h$ [3], [14]-[16], and for application, we take $U$ to be proportional to $h^{1 / 2}$ and $D$ to $h^{3 / 2}$, which are the appropriate scaling for a turbulent shallow-water flow over a smooth bed [17], [18].

\section{A. Semi-Infinite Flat Seabed}

For coastal areas where the variations in water depth becomes insignificant, we consider the highly simplified profile of a flat seabed with a constant water depth, $h=h_{0}$

(Fig. 1a), with a vertical beach at $y=0$. The advection-diffusion equation for the discharged effluent concentration in the far field $c_{f}(x, y)$ is

$h_{0} U_{0} \frac{\partial c_{f}}{\partial x}-h_{0} D_{0} \frac{\partial^{2} c_{f}}{\partial y^{2}}=Q \delta(x) \delta\left(y-\alpha h_{0}\right)$,

with the boundary condition $h_{0} D_{0} \frac{\partial c_{f}}{\partial y}=0$ at the shoreline $y=0$, and $c_{f}$ is assumed to be completely dissolved into the sea. $\delta(*)$ is the Dirac delta function used to represent the point at the end of outfall pipe where the discharge is made. Using dimensionless quantities

$y=Y h_{0}, x=X h_{0}$, and $c_{f}(x, y)=C_{f}(X, Y) Q / h_{0}{ }^{2} U_{0}$,

and for sufficiently long sea outfalls, the analytical solution of (1) for $X \geq 0$ is given by

$C_{f}=\sqrt{\frac{\lambda}{4 \pi X}} \exp \left(-\frac{\lambda(Y-\alpha)^{2}}{4 X}\right)$,

where the model parameter $\lambda=U_{0} h_{0} / D_{0}$ represents the effluent plume elongation in the $x$-direction [3], [9], [14], [15].
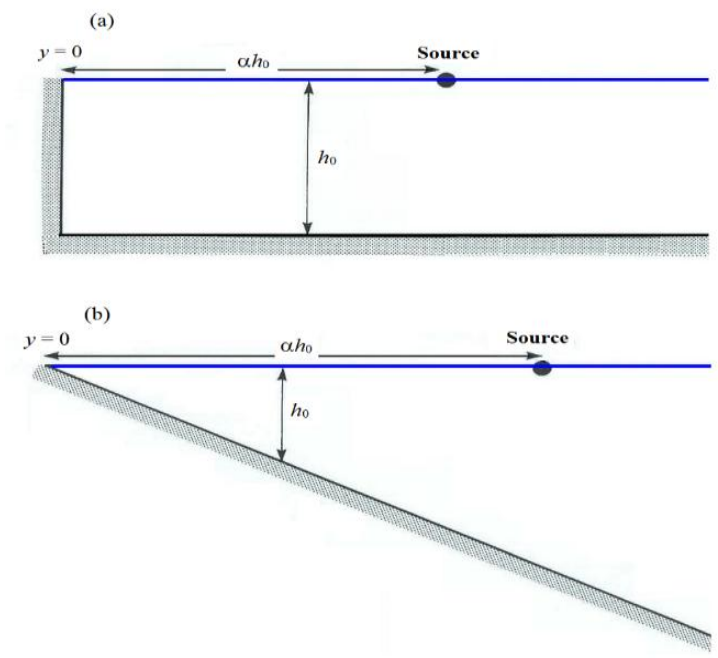

Fig. 1. Continuous seabed depth profile of a: (a) flat seabed and (b) sloping seabed 
Contours of concentration (2) for a point source at $\alpha=12$ were plotted in Fig. 2 for two values of $\lambda=0.1$ and $\lambda=0.2$ to illustrate the variability of sea conditions. The discharged effluent plumes appear to be spreading downstream parallel to the shoreline, and the larger the values of $\lambda$, the more elongated the plumes, which are mostly due to a stronger current $U_{0}$ with less longitudinal dispersivity $D_{0}$. The maximum concentration of 0.020 at the shoreline is shown by the blue contour.
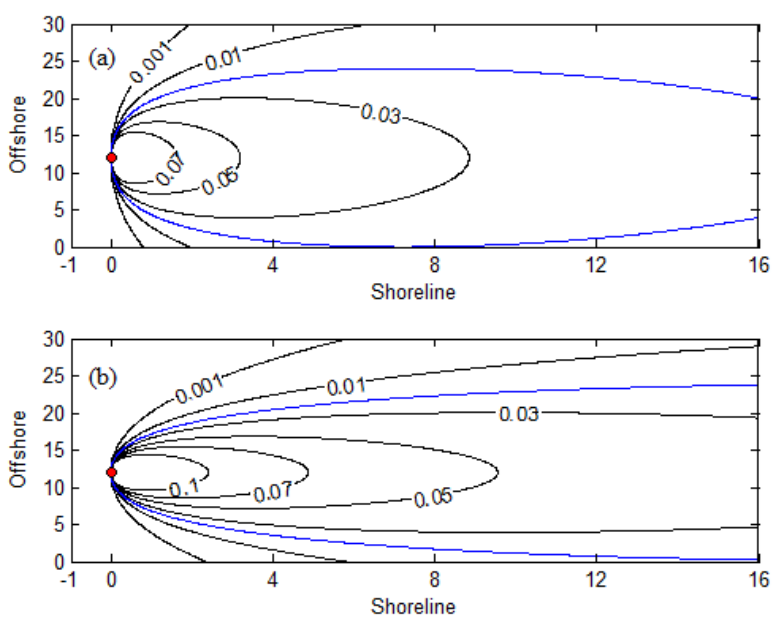

Fig. 2. Contours of concentration for a point source at $\alpha=12$ on a flat seabed for (a) $\lambda=0.1$ and (b) $\lambda=0.2$

The sensitive areas for assessing the environmental impact of effluent discharges into the sea would be along the coastal areas [3], [14]-[16]. Thus, on substituting $Y=0$ in concentration (2), and then by differentiating, it is straightforward to see that the maximum value of concentration at the shoreline is $C_{f \max }=1 / \alpha \sqrt{2 \pi e}$, which occurs at $X_{f \max }=\lambda \alpha^{2} / 2$.

Table- I: The RMZ for discharges on a flat seabed

\begin{tabular}{c|c|c|c}
\hline$\alpha$ & 11.5 & 12 & 12.5 \\
\hline$C_{* f}(\%)$ & 3.469 & 3.325 & 3.192 \\
\hline \hline$\lambda$ & \multicolumn{3}{|c}{$R_{* f}$} \\
\hline 0.050 & 3.306 & 3.600 & 3.906 \\
\hline 0.075 & 4.959 & 5.400 & 5.859 \\
\hline 0.100 & 6.613 & 7.200 & 7.813 \\
\hline 0.125 & 8.266 & 9.000 & 9.766 \\
\hline 0.150 & 9.919 & 10.800 & 11.719 \\
\hline 0.175 & 11.572 & 12.600 & 13.672 \\
\hline 0.200 & 13.225 & 14.400 & 15.625
\end{tabular}

Using the location of the maximum concentration at the shoreline, we proposed to set the size of the RMZ by $Y=\alpha$ and $X=R_{* f}=\lambda \alpha^{2} / 2$. From concentration (2), we obtain the concentration limit value at the edge of the RMZ as $C_{* f}=1 / \sqrt{2 \pi} \alpha$. The effect of uncertainty in sea conditions on the radius $R_{* f}$ is analyzed in Table I. Since $R_{* f}$ is linearly proportional with $\lambda$ and quadratically with $\alpha$, the value of $R_{* f}$ increases significantly for larger the values of $\lambda$. However, $C_{* f}$ is inversely proportional only with $\alpha$, and for $\alpha \geq 10$, the value of $C_{* f}<4 \%$. We conclude that the limiting concentration values and imposed radius of the mixing zone agreed with the recommended standard for conventional effluents discharge.

\section{B. Uniformly Sloping Seabed}

Coastal industrial plants are built predominantly on the sloping sandy beaches, where the water depth varies increasingly linear with the offshore distance $y$. If $m$ is the beach slope (Fig. 1b), then $h(y)=m y$. The advection-diffusion equation for the discharged effluent concentration in the far field $c_{s}(x, y)$ is

$\frac{\partial}{\partial x}\left(h U c_{s}\right)-\frac{\partial}{\partial y}\left(h D \frac{\partial c_{s}}{\partial y}\right)=Q \delta(x) \delta\left(y-\alpha h_{0}\right)$.

Similar to discharges on a flat seabed, the boundary condition is $h D \frac{\partial c_{s}}{\partial y}=0$ at the shoreline $y=0$, and $c_{s}$ is assumed to be completely dissolved into the sea. Assuming $U=U_{0} Y^{1 / 2}$ and $D=D_{0} Y^{3 / 2}$, in its dimensionless form, the analytical solution of (3) for $X \geq 0$ can be written as [3], [14], [15]

$C_{s}=\frac{\lambda}{m X}\left(\frac{1}{\alpha Y}\right)^{3 / 4} \exp \left(-\frac{\lambda(Y+\alpha)}{X}\right) I_{3 / 2}\left(\frac{2 \lambda \sqrt{\alpha Y}}{X}\right)$,

where $I_{3 / 2}(*)$ is a modified Bessel function of order $3 / 2$.
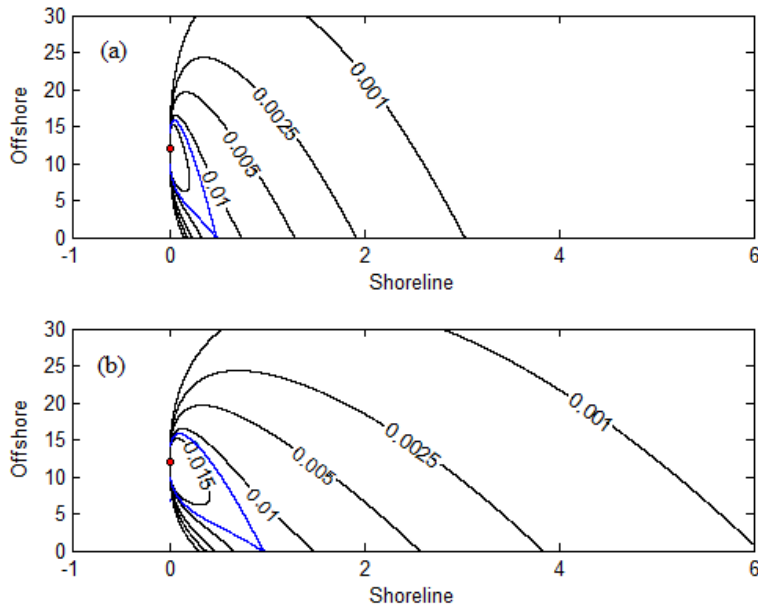

Fig. 3. Contours of concentration for a point source at $\alpha=12$ on a sloping seabed with $m=0.1$ for (a) $\lambda=0.1$ and (b) $\lambda=0.2$

Contours of concentration (4) were plotted in Fig. 3 with $m=0.1$ and $\alpha=12$ for two values of $\lambda=0.1$ and $\lambda=0.2$. Unlike discharges on a flat seabed, the discharged effluent plumes are spreading downstream and heading towards the shoreline, and 
cause a higher build up in concentration levels at the beach. The maximum concentration of 0.012 at the shoreline is shown by the blue contour.

Next, letting $Y \rightarrow 0$, and using the asymptotic expansion $I_{3 / 2}(z)=4(z / 2)^{3 / 2} / 3 \sqrt{\pi}$ in (4), we obtain an approximate effluent discharged concentration at the shoreline as $C_{s}(X, 0)=(4 / 3 m \sqrt{\pi})(\lambda / X)^{5 / 2} \exp (-\lambda \alpha / X)$. Next by differentiating, it has a maximum value of approximately $C_{s \max }=0.61 / m \alpha^{5 / 2}$, which occurs at $X_{s \max }=2 \lambda \alpha / 5$.

To formulate the RMZ, using the modified Bessel function by the asymptotic expansion $I_{3 / 2}(z)=\exp (z) / \sqrt{2 \pi z}$, and for sufficiently long sea outfalls, we first approximate concentration (4) as

$C_{s}=\frac{1}{m \alpha Y} \sqrt{\frac{\lambda}{4 \pi X}} \exp \left(-\frac{\lambda(\sqrt{Y}-\sqrt{\alpha})^{2}}{X}\right)$.

Then on setting $Y=\alpha$ and $X=R_{*_{s}}=2 \lambda \alpha / 5$, we obtain the concentration limit value at the edge of the RMZ as $C_{*_{s}}=\sqrt{5 / 8 \pi} / m \alpha^{5 / 2} . R_{*_{s}}$ is linearly proportional to both $\lambda$ and $\alpha$; whereas $C_{*_{s}}$ is inversely proportional to the slope $m$ and $\alpha$ to power 2.5 .

Table- II: The RMZ for discharges on a sloping seabed with $m=0.1$

\begin{tabular}{c|c|c|c}
\hline$\alpha$ & 11.5 & 12 & 12.5 \\
\hline$C_{*_{s}}(\%)$ & 0.995 & 0.894 & 0.807 \\
\hline \hline$\lambda$ & \multicolumn{3}{|c}{$R_{*_{s}}$} \\
\hline 0.050 & 0.230 & 0.240 & 0.25 \\
\hline 0.075 & 0.345 & 0.60 & 0.375 \\
\hline 0.100 & 0.460 & 0.480 & 0.500 \\
\hline 0.125 & 0.575 & 0.600 & 0.625 \\
\hline 0.150 & 0.690 & 0.720 & 0.750 \\
\hline 0.175 & 0.805 & 0.840 & 0.875 \\
\hline 0.200 & 0.920 & 0.960 & 1.000 \\
\hline 0.225 & 1.035 & 1.080 & 1.125 \\
\hline 0.250 & 1.150 & 1.200 & 1.250 \\
\hline
\end{tabular}

The variability in sea conditions on the radius $R_{*_{s}}$ are investigated in Table II for $m=0.1$. In comparison with discharges on a flat seabed, both the concentration limit $C_{*_{s}}$ and the radius $R_{*_{s}}$ are much smaller than that of the flat seabed's values. In fact, for calm sea conditions, the radius $R_{*_{s}}$ is less than a tenth of the value $R_{* f}$; and for $\alpha \geq 10$, the value of $C_{*_{S}}<1.5 \%$. This is due to the fact that the relatively low currents in the coastal areas delays the effluent transport towards the shore, whereas diffusion away from the shore continues to reduce the concentration [16], [17]. Thus, the larger the value of $\alpha$, the larger the distance travelled for the discharged effluent plumes to reach the shore (or the plumes do not feel the presence of the shoreline at $y=0)$.
Finally, we conclude that the imposed radius of the mixing zone and allowable concentration limits are suitable for the $\mathrm{RMZ}$ of acutely toxic coastal effluents discharge.

\section{Model Solutions for Coastal Discharges on a STEP SEABED DEPTH}

The seabed depth profile of eroding sandy beaches are changing as a result of coastal sediment transport [3], [12], [13]. Erosion is causing sediment particles to move alongshore and/or drift out to sea. As the sediment particles are deposited to the offshore region, a step beach profile is formed [13]. To account for the presence of an abrupt depth change in the profile (Fig. 4), we assume that the sudden water depth change is occurring only across the line $y=\ell h_{0}$ (parallel to the straight shoreline at $y=0$ ), where $r=h_{1} / h_{0}$ is the ratio of water depths across the discontinuity line $y=\ell h_{0}$. By writing the discharged effluent concentration in the far-field

$c(x, y)=\left\{\begin{array}{lc}c_{0}(x, y), & 0 \leq y<\ell h_{0} \\ c_{1}(x, y), & y \geq \ell h_{0}\end{array}\right.$,

the solution of the advection-diffusion equation can be obtained using the method of image [14], where the depth discontinuity at the line $y=\ell h_{0}$ will be considered as a (reflecting or absorbing) barrier (Fig. 5), beyond which the water depth is zero (or infinite).
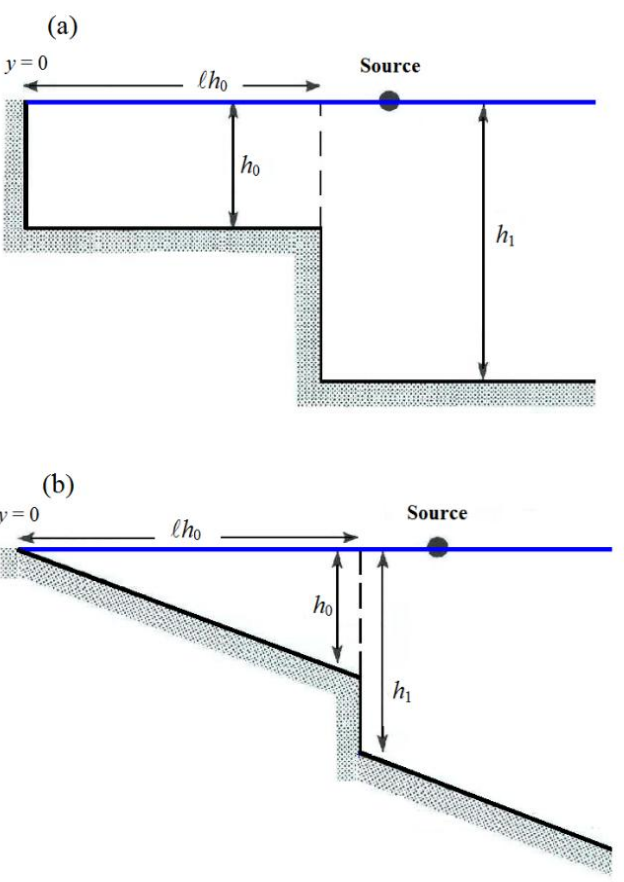

Fig. 4. Discontinuous step seabed depth profile in a: (a) flat seabed and (b) sloping seabed

To make use of the presence of shallow water depth, we consider the case where the effluent discharge is made from a point source at $\left(x=0, y=\alpha_{1} h_{0}\right)$ with $\alpha_{1}>\ell$, i.e. the effluent is released in the deeper offshore region 
$y \geq \ell h_{0}$ at a distance $\alpha_{1} h_{0}$ from the shoreline (Fig. 5). Treating the discontinuity line $y=\ell h_{0}$ as a reflecting boundary, the solution $c_{1}(x, y)$ is obtained due to a point source at $\left(0, \alpha_{1} h_{0}\right)$ discharging with a rate $Q$ and an image source at $\left(0,\left(2 \ell-\alpha_{1}\right) h_{0}\right)$ discharging with a rate $a Q$. In the shallow nearshore region $0 \leq y<\ell h_{0}$, the line $y=\ell h_{0}$ is treated as an absorbing boundary, and the solution $c_{0}(x, y)$ is obtained due to a virtual source at $\left(0, \beta h_{0}\right)$ discharging with a rate $b Q$, where $\beta>\ell$. However, as there can be no discontinuities in either the effluent concentration or its gradient across the discontinuity line $y=\ell h_{0}$, the matching conditions to be satisfied are

$\lim _{y \rightarrow \ell h_{0}} c_{0}=\lim _{y \rightarrow \ell h_{0}} c_{1}$

and

$$
\lim _{y \rightarrow \ell h_{0}} h_{0} D_{0} \frac{\partial c_{0}}{\partial y}=\lim _{y \rightarrow \ell h_{0}} h_{1} D_{1} \frac{\partial c_{1}}{\partial y} .
$$

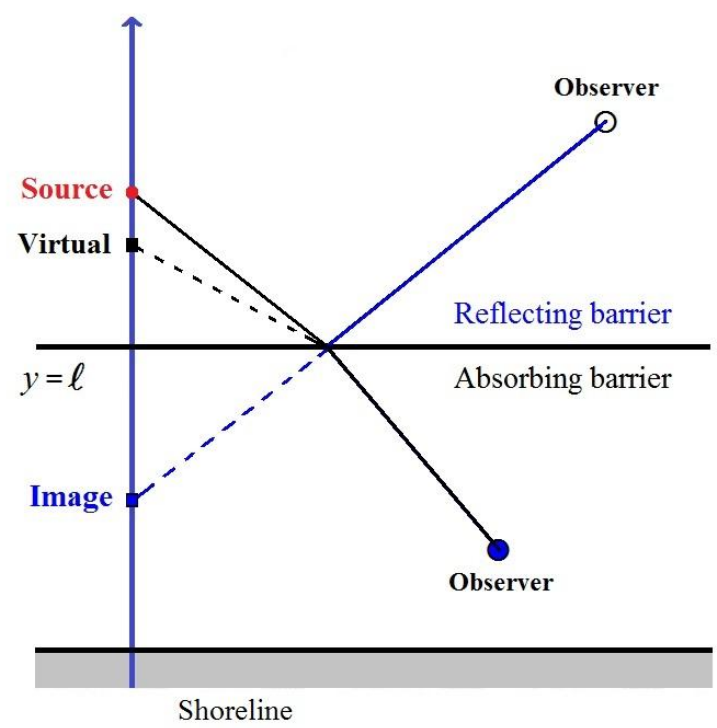

Fig. 5. Plan view of the method of image for a point source in the deeper region $y \geq \ell h_{0}$

\section{A. Flat-Step Seabed}

For a step discontinuity on a flat seabed depth profile (Fig. 4a), the water depth $h_{f}$ varies according to

$h_{f}(y)=\left\{\begin{array}{cc}h_{0}, & 0 \leq y<\ell h_{0} \\ h_{1}, & y \geq \ell h_{0}\end{array}\right.$.

Note that if $r=1$, there is no depth change across the discontinuity line $y=\ell h_{0}$, and this flat-step depth profile is exactly that of a simple flat seabed.

Similar to the previous section on a flat seabed, the advection-diffusion equation for concentration $c_{f 0}(x, y)$ in the nearshore region $0 \leq y<\ell h_{0}$ is given by (1) but with a virtual point source at $\left(0, \beta_{f} h_{0}\right)$ discharging with a rate $b_{f} Q$, and thus the solution, in its dimensionless form, is
$C_{f 0}=b_{f} \sqrt{\frac{\lambda}{4 \pi X}} \exp \left(-\frac{\lambda\left(Y-\beta_{f}\right)^{2}}{4 X}\right)$

In the deeper offshore region $y \geq \ell h_{0}$, on applying the superposition principle, the advection-diffusion equation for concentration $c_{f 1}(x, y)$ is

$$
\begin{aligned}
h_{1} U_{1} \frac{\partial c_{f 1}}{\partial x}-h_{1} D_{1} \frac{\partial^{2} c_{f 1}}{\partial y^{2}}=Q \delta(x) \delta\left(y-\alpha_{1} h_{0}\right) \\
+a_{f} Q \delta(x) \delta\left(y-2 \ell h_{0}+\alpha_{1} h_{0}\right)
\end{aligned}
$$

and the solution, in its dimensionless form, is

$$
\begin{aligned}
C_{f 1}=\frac{1}{r^{2}} \sqrt{\frac{\lambda}{4 \pi X}} & {\left[\exp \left(-\frac{\lambda\left(Y-\alpha_{1}\right)^{2}}{4 r X}\right)\right.} \\
+ & \left.a_{f} \exp \left(-\frac{\lambda\left(Y-2 \ell+\alpha_{1}\right)^{2}}{4 r X}\right)\right]
\end{aligned}
$$

where $U_{1}=U_{0} r^{1 / 2}$ and $D_{1}=D_{0} r^{3 / 2}$. The dimensionless form of the matching conditions

$\lim _{Y \rightarrow \ell} C_{f 0}=\lim _{Y \rightarrow \ell} C_{f 1}$ and $\lim _{Y \rightarrow \ell} \frac{\partial C_{f 0}}{\partial Y}=\lim _{Y \rightarrow \ell} r^{5 / 2} \frac{\partial C_{f 1}}{\partial Y}$

may now be applied in order to calculate $a_{f}, b_{f}$ and $\beta_{f}$, and we obtain

$$
\beta_{f}=\ell+\frac{\alpha_{1}-\ell}{\sqrt{r}}, a_{f}=\frac{r^{2}-1}{r^{2}+1} \text { and } b_{f}=\frac{2}{r^{2}+1} .
$$

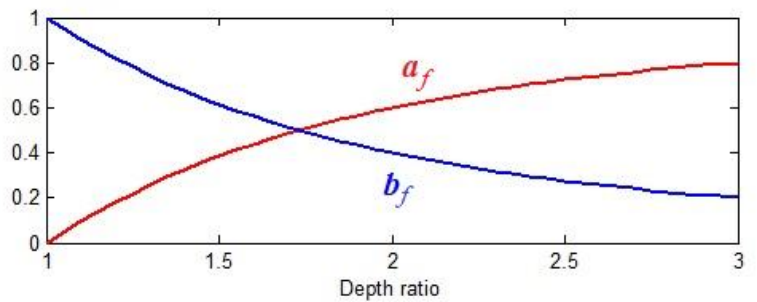

(b)

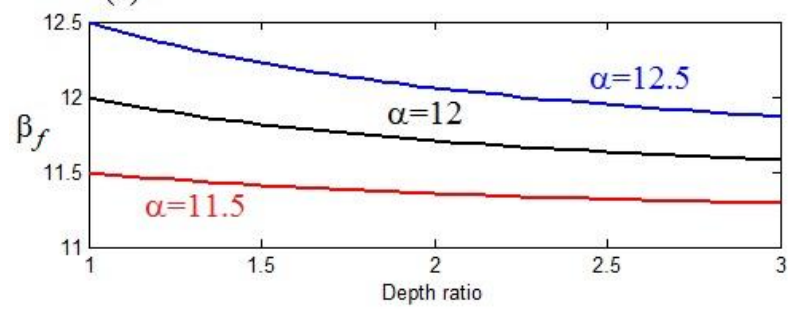

Fig. 6. A flat-step seabed when $\ell=11$ : (a) the coefficients

$$
a_{f} \text { and } b_{f} ; \text { and (b) } \beta_{f}
$$

It is easy to verify, $a_{f}+b_{f}=1$ and if there is no depth change, i.e. $r=1$, then $\beta_{f}=\alpha_{1}, a_{f}=0$ and $b_{f}=1$. The variations of these coefficients for $r \geq 1$ when $\ell=11$ are shown in Fig. 6. The value of $a_{f}$ is increasing $0 \leq a_{f}<1$, whereas the value of $b_{f}$ is decreasing $0<b_{f} \leq 1$. The 
value of $\beta_{f}$ is also decreasing $\ell<\beta_{f} \leq \alpha_{1}$.

To calculate the RMZ, by substituting $Y=\alpha_{1}$ and $X=R_{*} f 1=\lambda \beta_{f}^{2} / 2$ in concentration (5), we obtain the concentration limit at the edge of the RMZ as

$C_{* f 1}=\frac{1}{\sqrt{2 \pi} \beta_{f} r^{2}}\left[1+\frac{r^{2}-1}{r^{2}+1} \exp \left\{-2\left(1-\frac{\ell}{\beta_{f}}\right)^{2}\right\}\right]$.

In comparison with discharges on a flat seabed, we obtain $\frac{R_{* f 1}}{R_{* f}}=\left(\frac{\beta_{f}}{\alpha_{1}}\right)^{2}$

and

$$
\frac{C_{* f 1}}{C_{* f}}=\frac{\alpha_{1}}{\beta_{f} r^{2}}\left[1+\frac{r^{2}-1}{r^{2}+1} \exp \left\{-2\left(1-\frac{\ell}{\beta_{f}}\right)^{2}\right\}\right] .
$$

Since $\beta_{f} \leq \alpha_{1}$ for $r \geq 1$, the radius $R_{* f 1} \leq R_{* f}$ and the concentration limit $C_{* f 1} \leq C_{* f}$.

The reductions in the concentration limit $C_{* f 1}$ and in the ratio of radius $R_{* f 1} / R_{* f}$ for $r \geq 1$ on a flat-step seabed when $\ell=11$ are shown in Fig. 7 for three values of $\alpha_{1}=11.5,12$ and 12.5. In particular for the case of $\alpha_{1}=12$, a reduction in value of $C_{* f 1}$ (relative to $C_{* f}$ ) is about $38 \%$ for $r=1.5$ to about $72 \%$ for $r=2.5$. However, the reduction in $R_{* f 1}$ (relative to $R_{* f}$ ) is less significant, and it is only about $5 \%$ for $r=2$ to about $7 \%$ for $r=3$.

(a)

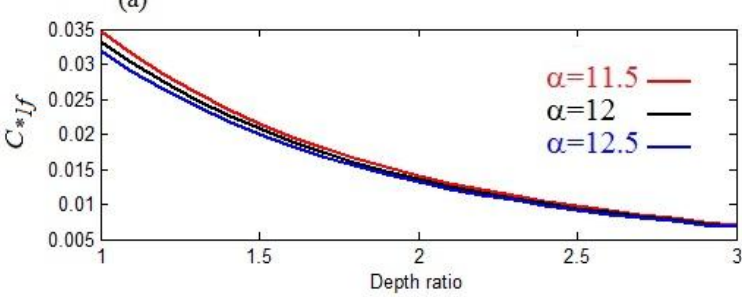

(b)

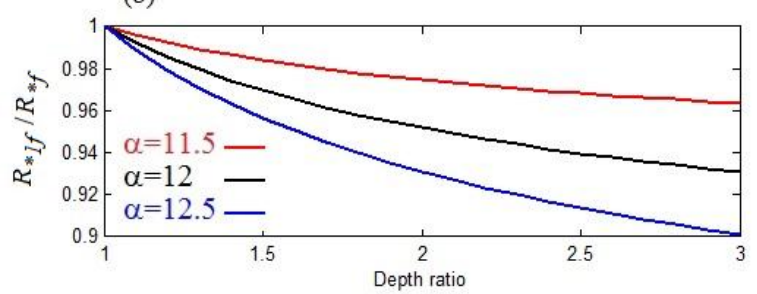

Fig. 7. The RMZ for discharges on a flat-step seabed when $\ell=11$ : (a) the concentration limit $C_{* f 1}$ and (b) the ratio of radius $R_{* f 1} / R_{* f}$

\section{B. Slope-Step Seabed}

For a step discontinuity on a sloping seabed depth profile (Fig. 4b), the water depth $h_{s}$ varies as

$h_{s}(y)=\left\{\begin{array}{cc}m_{0} y, & 0 \leq y<\ell h_{0} \\ m_{1} y, & y \geq \ell h_{0}\end{array}\right.$,

where the slope in the nearshore region $m_{0}=1 / \ell$ and in the offshore region $m_{1}=r / \ell=r m_{0}$. Note that if $r=1$, there is no depth change across the discontinuity line $y=\ell h_{0}$, and this slope-step depth profile is exactly that of a uniformly sloping seabed.

Similar to the previous section on a sloping seabed, the advection-diffusion equation for concentration $c_{s 0}(x, y)$ in the nearshore region $0 \leq y<\ell h_{0}$ is given by (3) but with a virtual point source at $\left(0, \beta_{s} h_{0}\right)$ discharging with a rate $b_{s} Q$, and thus the solution, in its dimensionless form, is

$C_{s 0}=\frac{b_{s} \lambda}{m_{0} X}\left(\frac{1}{\beta_{s} Y}\right)^{3 / 4} \exp \left(-\frac{\lambda\left(Y+\beta_{s}\right)}{X}\right) I_{3 / 2}\left(\frac{2 \lambda \sqrt{\beta_{s} Y}}{X}\right)$.

Next, on applying the superposition principle, the advection-diffusion equation for concentration $c_{s 1}(x, y)$ in the deeper offshore region $y \geq \ell h_{0}$ is

$$
\begin{aligned}
h_{1} U_{1} \frac{\partial c_{s 1}}{\partial x}-\frac{\partial}{\partial y}\left(h_{1} D_{1} c_{s 1}\right) & =Q \delta(x) \delta\left(y-\alpha_{1} h_{0}\right) \\
+ & a_{s} Q \delta(x) \delta\left(y-2 \ell h_{0}+\alpha_{1} h_{0}\right)
\end{aligned},
$$

and the solution, in its dimensionless form, is

$$
\begin{aligned}
& C_{s 1}=\frac{\lambda}{m_{1} r^{3 / 2} X} \\
& {\left[\left(\frac{1}{\alpha_{1} Y}\right)^{3 / 4} \exp \left(-\frac{\lambda\left(Y+\alpha_{1}\right)}{r X}\right) I_{3 / 2}\left(\frac{2 \lambda \sqrt{\alpha_{1} Y}}{r X}\right)\right.} \\
& +a_{s}\left(\frac{1}{\left(2 \ell-\alpha_{1}\right) Y}\right)^{3 / 4} \exp \left(-\frac{\lambda\left(Y+2 \ell-\alpha_{1}\right)}{r X}\right) \\
& \left.\quad \times I_{3 / 2}\left(\frac{2 \lambda \sqrt{\left(2 \ell-\alpha_{1}\right) Y}}{r X}\right)\right]
\end{aligned}
$$

where $U_{1}=U_{0} r^{1 / 2} Y^{1 / 2}$ and $D_{1}=D_{0} r^{3 / 2} Y^{3 / 2}$. Note that the arguments of modified Bessel functions are approximately the same provided $2 \ell-\alpha_{1} \approx \alpha_{1}$, i.e. when $\alpha_{1} / \ell \approx 1$.

After approximating the modified Bessel functions, the matching conditions, in its dimensionless form,

$$
\lim _{Y \rightarrow \ell} C_{s 0}=\lim _{Y \rightarrow \ell} C_{s 1}
$$

and

$$
\lim _{Y \rightarrow \ell} m_{0} Y^{5 / 2} \frac{\partial C_{s 0}}{\partial Y}=\lim _{Y \rightarrow \ell} m_{1} r^{3 / 2} Y^{5 / 2} \frac{\partial C_{s 1}}{\partial Y}
$$

may now be applied in order to calculate $a_{s}, b_{s}$ and $\beta_{s}$, we obtain

$$
\sqrt{\beta_{s}}=\sqrt{\ell}+\frac{\sqrt{\alpha_{1}}-\sqrt{\ell}}{\sqrt{r}}, a_{s}=\left(\frac{2 \ell-\alpha_{1}}{\alpha_{1}}\right)\left(\frac{r^{2}-1}{r^{2}+1}\right)
$$

and 


$$
b_{s}=\left(\frac{\beta_{s}}{\alpha_{1}}\right)\left(\frac{2}{r^{2}+1}\right) \text {. }
$$

Unlike discharges on a flat-step seabed, both coefficients $a_{s}$ and $b_{s}$ are dependent of $\alpha_{1}$. If there is no depth change, of these coefficients for $r \geq 1$ when $\ell=11$ are shown in Fig. 8. For $\alpha_{1}=12$, similar to that of the flat-step seabed, the value of $a_{s}$ is increasing $0 \leq a_{s}<1$; while the value of $b_{s}$ is decreasing $0<b_{s} \leq 1$. The value of $\beta_{s}$ is also decreasing $\ell<\beta_{s} \leq \alpha_{1}$.

Following the previous section on a sloping seabed, using the modified Bessel function by the asymptotic expansion, we approximate concentration (6) as

$$
\begin{array}{r}
C_{s 1}=\frac{1}{m_{1} r \alpha_{1} Y} \sqrt{\frac{\lambda}{4 \pi X}}\left[\exp \left(-\frac{\lambda\left(\sqrt{Y}-\sqrt{\alpha_{1}}\right)^{2}}{r X}\right)\right. \\
\left.+\frac{a_{s} \alpha_{1}}{2 \ell-\alpha_{1}} \exp \left(-\frac{\lambda\left(\sqrt{Y}-\sqrt{2 \ell-\alpha_{1}}\right)^{2}}{r X}\right)\right]^{.}
\end{array}
$$

(a)

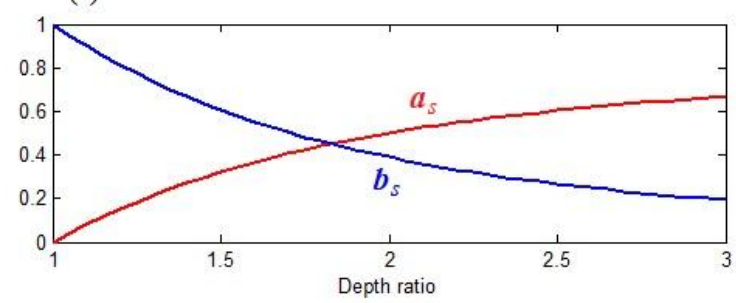

(b)

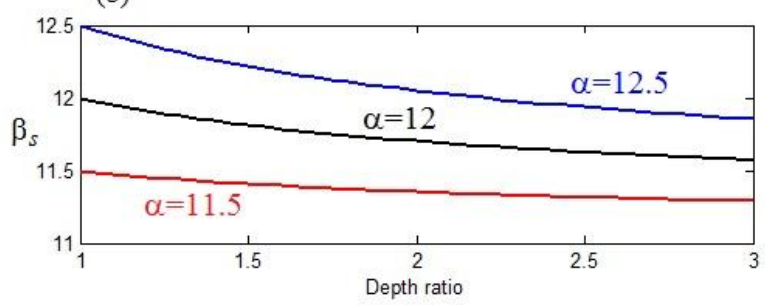

Fig. 8. A slope-step seabed when $\ell=11$ : (a) the coefficients

$$
a_{s} \text { and } b_{s} \text { (for } \alpha_{1}=12 \text { ); and (b) } \beta_{s}
$$

Then, by substituting $Y=\alpha_{1}$ and $X=R_{* s 1}=2 \lambda \beta_{s} / 5$, we obtain the concentration limit at the edge of the RMZ as

$$
\begin{aligned}
C_{*_{s} 1}= & \sqrt{\frac{5}{8 \pi}} \frac{\ell}{\beta_{s}\left(r \alpha_{1}\right)^{2}}[1+ \\
& \left.\frac{r^{2}-1}{r^{2}+1} \exp \left\{-5\left(\frac{\ell-\sqrt{\left(2 \ell-\alpha_{1}\right) \alpha_{1}}}{r \beta_{s}}\right)\right\}\right]
\end{aligned}
$$

In comparison with discharges on a sloping seabed, we obtain

$\frac{R_{*_{S} 1}}{R_{*_{s}}}=\frac{\beta_{s}}{\alpha_{1}}$ i.e. $r=1$, then $\beta_{s}=\alpha_{1}, a_{s}=0$ and $b_{s}=1$. The variations

and

$$
\frac{C_{*_{s} 1}}{C_{*_{s}}}=\frac{1}{r^{2}} \sqrt{\frac{\alpha_{1}}{\beta_{s}}}\left[1+\frac{r^{2}-1}{r^{2}+1} \exp \left\{-5\left(\frac{\ell-\sqrt{\left(2 \ell-\alpha_{1}\right) \alpha_{1}}}{r \beta_{s}}\right)\right\} .\right.
$$

We note that the radius $R_{*_{s} 1}$ is exactly the same to that of discharges on a sloping seabed radius $R_{*_{S}}$ (with a virtual source at $\left.\left(0, \beta_{s} h_{0}\right)\right)$. Thus, the presence of a step water depth on a sloping seabed does not change the radius $R_{*_{s}}$. For $r \geq 1$, the concentration limit $C_{*_{s} 1}$ is smaller than that of discharges on a sloping seabed $C_{*_{s}}$.

The concentration limit $C_{*_{s} 1}$ for discharges on a slope-step seabed when $\ell=11$ are shown in Fig. 9 for three values of $\alpha_{1}=11.5,12$ and 12.5 . The value $C_{*_{s} 1}$ is less than $1.5 \%$ for $r \geq 1$. Similar to a flat-step seabed, for the case of $\alpha_{1}=12, C_{* s 1}=0.0098$ for $r=1$, and as $r$ increases, the reduction in the concentration limit increases, from about $38 \%$ for $r=1.5$ to more than $72 \%$ for $r=2.5$. However, the reduction in $R_{* s 1}$ is much smaller; of about $2.5 \%$ for $r=2$ to about $3.6 \%$ for $r=3$.

(a)

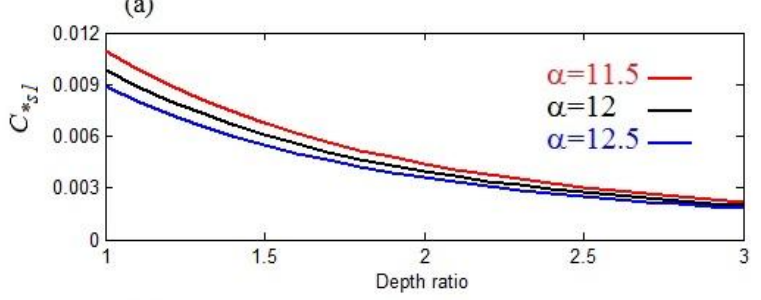

(b)

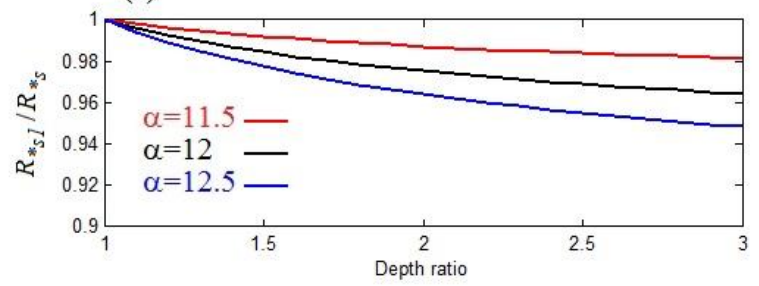

Fig. 9. The RMZ for discharges on a slope-step seabed when $\ell=11$ : (a) the concentration limit $C_{*_{s} 1}$ and (b) the ratio of radius $R_{* s 1} / R_{* s}$

\section{CONCLUDING REMARKS}

The effect of the seabed depth profile upon mixing and spreading of effluent wastewater continuously being discharged into the sea is investigated analytically using simple models of a flat seabed with a constant water depth and a uniformly sloping seabed. Using the location of the maximum value of concentration at the shoreline, the radius of the RMZ and the concentration limit at the end of the RMZ are formulated. These model values are found to have agreed with the recommended size of a mixing zone imposed and implemented in practice by the regulatory authorities to control coastal effluent discharges from marine outfall systems [7], [10]. The radius of the RMZ obtained for a sloping seabed is less than a

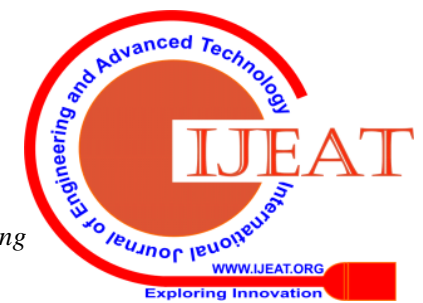


tenth of that value for a flat seabed, and thus, it is suitable for the RMZ of acutely toxic coastal effluents discharge. To improve the models and to illustrate the effect of shallow water depth in the nearshore region, a step discontinuity in depth profile is then introduced.

The use of mathematical models has been a key strategy for assessing the potential environmental impacts of outfall effluent discharges. While the far field modelling in this paper involves drastic simplifications, key physical mixing and dispersion processes are represented, and thus the analytical solution remains useful in providing a qualitative understanding and in suggesting general behaviour of the effluent discharge plumes in coastal waters [19], [20]. The models can be applied to any type of pollutant continuously being discharged into the sea from a marine outfall system; for example, treated effluents from coastal sewage works [1], [2], toxic contaminants from industrial installations in coastal areas [2], hot water discharged from coastal power stations [21] and brine discharge from desalination plants [4], [7].

\section{REFERENCES}

1. P. J. W. Roberts, H. J. Salas, F. M. Reef, M. Libhaber, A. Labe, and J. C. Thomson, Marine Wastewater Outfalls and Treatment Systems. International Water Association Publishing, London, 2010.

2. Institution of Civil Engineers, Long Sea Outfalls. Thomas Telford, Ltd., London, 1989.

3. A. Purnama, H. A. Al Maamari, and E. Balakrishnan, "Optimal outfall systems for nearshore effluent discharges on eroded sandy beaches," Journal of Coupled Systems and Multiscale Dynamics, vol. 5, no. 2-4, 2017, pp. 217-224

4. A. Purnama, H. H. Al-Barwani, T. Bleninger, and R. L. Doneker, "CORMIX simulations of brine discharges from Barka plants, Oman," Desalination and Water Treatment, vol. 32, 2011, pp. 329-338.

5. G. H. Jirka, T. Bleninger, R. Burrows, and T. Larsen, "Environmental quality standards in the EC-Water Framework Directive: Consequences for water pollution control for point sources," European Water Management Online (EWMO), 2004. Available: www.ewaonline.de

6. US EPA, Review of the D-CORMIX Model. Science Advisory Board Report EPA-SAB-EC-99-011, Washington DC, USA, 1999.

7. S. A. Jenkins, J. D. Paduan, P. J. W. Roberts, D. Schlenk, and J. S. Weis, Management of Brine Discharges to Coastal Waters Recommendations of a Science Advisory Panel. Technical Report 694, California Water Resources Board, California, USA, 2012.

8. A. Purnama, "Environmental quality standards for brine discharge from desalination plants," In M. S. Baawain, B. S. Choudri, M. Ahmed, A. Purnama (Eds.) Recent Progress in Desalination, Environmental and Marine Outfall Systems, Springer series on Environment, 2015, Chapter 18, pp. 257-268.

9. A. Purnama, "Modeling dispersion of brine discharges from multiple desalination outfalls," In O. Abdallah, A. Kacimov, M. Chen, A. Al-Maktoumi, T. K. S. Al-Hosni, I. Clark (Eds.) Water Resources in Arid Areas: The Way Forward, Springer series on Water, 2017, Chapter 19, pp. 335-349.

10. N. Ahmad, and R.E. Baddour, "A review of sources, effects, disposal methods, and regulations of brine into marine environments," Ocean and Coastal Management, vol. 87, 2014, pp. 1-7.

11. A. M. J. Ragas, J. L. M. Haans, and R. S. E. W. Leuven, "Selecting water quality models for discharge permitting," European Water Pollution Control, vol. 7, 1997, pp. 59-67.

12. J. Hardisty, Beaches Form and Processes. Unwin Hyman Ltd., London, 1990.

13. H. Wang, "Beach profile modelling," in R.A. Dalrymple (ed.) Physical Modelling in Coastal Engineering, A. A. Balkema, Rotterdam, Netherlands, 1985, pp. 237-271.

14. A. Kay, "The effect of cross-stream depth variations upon contaminant dispersion in a vertically well-mixed current," Estuarine, Coastal and Shelf Science, vol. 24, 1987, pp. 177-204.

15. H. H. Al-Barwani, and A. Purnama, "Analytical solutions for brine discharge plumes on a sloping beach," Desalination and Water Treatment, vol. 11, 2009, pp. 2-6.
16. R. Smith, "Effects of non-uniform currents and depth variations upon steady discharges in shallow water," Journal of Fluid Mechanics, vol. 110, 1981, pp. 373-380.

17. R. Smith, "The dependence of shoreline contaminant levels upon the siting of an effluent outfall," Journal of Fluid Mechanics, vol. 130, 1983, pp. 153-164.

18. D. W. Ostendorf, "Longshore dispersion over a flat beach," Journal of Geophysical Research, vol. 87, 1982, pp. 4241-4248.

19. D. A. Chin, and P. J. W. Roberts, "Model of dispersion in coastal waters," Journal of Hydraulic Engineering, vol. 111, 1985, pp. 12-28.

20. I. R. Wood, "Asymptotic solutions and behavior of outfall plumes," Journal of Hydraulic Engineering, vol. 119, 1993, pp. 553-580.

21. J. F. Macqueen, and R. W. Preston, "Cooling water discharges into a sea with a sloping bed,” Water Research, vol. 17, 1983, pp. 389-395.

\section{Authors Profile}

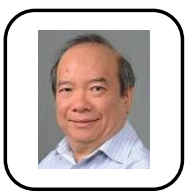

Anton Purnama, Associate Professor of the Department of Mathematics, College of Science, Sultan Qaboos University (SQU), Muscat, Oman. He obtained his BA (in Mathematics) and PhD from (DAMTP of) University of Cambridge, UK; and before joining at SQU, he worked in the UK at the NERC Water Resource Systems Research Unit, Department of Civil Engineering, University of Newcastle upon Tyne, and at the Department of Mathematical Sciences, Loughborough University.

His current research interests is focused on mathematical models for coastal effluent discharges from marine outfall systems and sediment transport in the far-field. He involves in consultancy work for a number of marine outfall systems in Oman, and is member of the Steering Committee, Water Research Center at SQU. He is also a member of the International Association for Hydro-Environment Engineering and Research (IAHR) and was a member of the Leadership Team of the IAHR/IWA Joint Committee on Marine Outfall Systems.

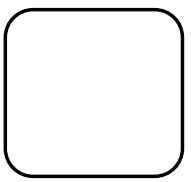

Huda A. Al-Maamari, PhD student at Department of Mathematics, College of Science, Sultan Qaboos University (SQU), Muscat, Oman. She obtained her BSc (in Mathematics) and MSc (in Applied Mathematics) from Department of Mathematics and Statistics, SQU. She is Oman. currently on study leave from the Ministry of Education,

Her research interests are on the analytical model studies for spreading and dispersion of coastal effluent discharges through marine outfall systems to investigate the effect of variations of seabed depth profiles, include change due to beach erosion, and the effect of variability of discharged effluents decay with water depth

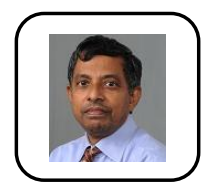

Easwaran Balakrishnan, Associate Professor, Department of Mathematics, College of Science, Sultan Qaboos University (SQU), Muscat, Sultanate of Oman. He obtained his MSc (in Mathematics) from University of Canterbury, New Zealand; and $\mathrm{PhD}$ from Massey University, New Zealand.

His other research interests include finding critical ambient and storage temperatures in spontaneous combustion models, predator-prey interactions in mathematical biology, bioreactors and computational mathematics. 